\title{
ON THE REMOVABLE SINGULARITIES FOR MEROMORPHIC MAPPINGS
}

\author{
E. M. CHIRKA
}

\begin{abstract}
If $E$ is a closed subset of locally finite Hausdorff $(2 n-2)$-measure on an $n$-dimensional complex manifold $\Omega$ and all the points of $E$ are nonremovable for a meromorphic mapping of $\Omega \backslash E$ into a compact Kähler manifold, then $E$ is a pure $(n-1)$-dimensional complex analytic subset of $\Omega$.
\end{abstract}

1. This paper was inspired by the following question of E. L. Stout $[\mathbf{S}]$ : Let $E$ be a closed subset of the complex projective space $\mathbb{P}^{n}, n \geq 2$, such that the Hausdorff ( $2 n-2)$-measure of $E$ with respect to the Fubini-Study metric is less than that of a complex hyperplane in $\mathbb{P}^{n}$. Is it then true that $E$ is a set of removable singularities for meromorphic functions? Using natural projections of $\mathbb{P}^{n}$ onto hyperplanes G. Lupacciolu $[\mathbf{L u}]$ has shown the removability of $E$ under additional conditions on $E$. A complete affirmative solution of the problem was given by M. Lawrence $[\mathbf{L a}]$. In this paper we consider an extension of the problem for meromorphic mappings on an arbitrary complex manifold and solve it (for special target manifolds) using the Oka-Nishino Theorem $[\mathbf{N}]$ on pseudoconcave sets.

There are two general notions of meromorphic mapping between complex manifolds $M, X$.

A meromorphic map in the sense of Remmert $[\mathbf{R}]$ (or strongly meromorphic map) is a holomorphic map $f: M \backslash A \rightarrow X$ defined in the complement of an analytic subset $A \subset M$ and such that the closure of its graph is an analytic subset of $M \times X$ with proper projection $\pi$ in $M$ (the last property is satisfied automatically if the target manifold $X$ is compact). It follows easily from the definition that the set

Supported by Russian Fund of Fundamental Researches, project No 96-01-00530. 
$A_{f}=\left\{z \in M: \operatorname{dim} \pi^{-1}(z)>0\right\}$ is an analytic subset of (complex) codimension at least two and $f$ extends holomorphically to $M \backslash A_{f}$.

A meromorphic map in the sense of Stoll [St] (or weakly meromorphic map) is a holomorphic map $f: M \backslash A \rightarrow X$, where $A$ is an analytic subset of codimension larger than or equal to $M$, such that, for any analytic disk $\varphi: \Delta \rightarrow M$ with $\varphi(\Delta) \not \subset A$, the restriction $f \circ \varphi: \Delta \backslash \varphi^{-1}(A) \rightarrow X$ extends holomorphically to the disc $\Delta$.

We proceed now to state our main result. We point out that there is no difference between strongly and weakly meromorphic maps as far as its statement and proof are concerned, and thus we use the term "meromorphic" to mean always "strongly meromorphic" or always "weakly meromorphic".

Theorem. Let $E$ be a closed subset of locally finite Hausdorff $(2 n-2)$-measure on an $n$-dimensional complex manifold $\Omega$ and let $f$ be a meromorphic map from $\Omega \backslash E$ into a complex manifold $X$. If $X$ has the meromorphic extension property and $E$ does not contain any $(n-1)$-dimensional closed analytic subset of $\Omega$, then $f$ extends to a meromorphic mapping from $\Omega$ into $X$.

Here we say that $X$ has the meromorphic extension property, if every meromorphic mapping $\varphi: T \rightarrow X$ on the "squeezed polydisc"

$T=\left\{(z, w) \in \mathbb{C}_{z}^{n-1} \times \mathbb{C}_{w}:|z|<r,|w|<1\right.$ or $\left.|z|<1,1-r<|w|<1\right\}$,

$0<r<1, n \geq 2$, extends to a meromorphic mapping $\tilde{\varphi}: U \rightarrow X$ on the unit polydisc $U:|z|<1,|w|<1$. By a result of $\mathrm{S}$. M. Ivashkovich $[\mathbf{I}]$ every compact Kähler manifold $X$ has the meromorphic extension property, so we have a lot of nice examples of such $X$.

The case of meromorphic functions $\left(X=\mathbb{P}^{1}\right)$ is almost trivial in the consideration: every meromorphic function in a squeezed polydisc is represented as a ratio of two holomorphic functions (see $[\mathbf{K S}]$ ) and thus it extends meromorphically into $U$. So the answer on the question of Stout is affirmative because the set $E$ in the question can not contain any complex analytic subset of $\mathbb{P}^{n}$. (By Chow Theorem such a set is algebraic, hence, its Hausdorff $(2 n-2)$-measure is not less than the measure of a complex hyperplane (see, e.g., $[\mathbf{C}]$ ).)

Note also that the meromorphic extension claimed in the Theorem is not holomorphic in general for holomorphic mappings $f: \Omega \backslash E \rightarrow X$, even for $X=\mathbb{P}^{1}$ (one can take a meromorphic map $f: \Omega \rightarrow X$ with nonempty set $A_{f}$ of indeterminacy of $f$ and put $E=A_{f}$ ). 
2. Here we prove the Theorem.

Let $E^{\prime}$ be the set of points $a \in E$ such that $f$ extends meromorphically into a neighbourhood of $a$. Then $S:=E \backslash E^{\prime}$ is closed. As the complement of $E$ is locally connected in $\Omega$, these local meromorphic extensions of $f$ to points of $E^{\prime}$ glue together into the unique meromorphic mapping $f: \Omega \backslash S \rightarrow X$ (we keep the notation $f$ ).

The proof of the Theorem is accomplished now in two steps. First we prove that $S$ is locally pseudoconcave (Lemma 1), and then we prove that $S$ is complex analytic (Lemma 2). (A closed subset $\Sigma$ of a complex manifold is called locally pseudoconcave if for every point $a \in \Sigma$ there exists a Stein neighborhood $V$ of $a$ such that $V \backslash \Sigma$ is Stein.) It follows then from the condition of the Theorem that $S$ is empty, and hence, $f$ extends meromorphically into $\Omega$.

Lemma 1. $S$ is locally pseudoconcave in $\Omega$.

Proof: Let $a \in S$, let $V$ be a neighborhood of $a$ which is biholomorphic to a ball in $\mathbb{C}^{n}$ and let $\varphi: T \rightarrow V \backslash S$ be a holomorphic embedding. Then $\varphi$ extends to a holomorphic embedding $\tilde{\varphi}: U \rightarrow V$ (see [DG]) because $V$ is Stein. As $X$ has the meromorphic extension property, the meromorphic map $f \circ \varphi: T \rightarrow X$ extends to a meromorphic map of $U$ into $X$ and thus $f$ extends meromorphically into the domain $\tilde{\varphi}(U) \subset V$. By the definition of $S, \tilde{\varphi}(U)$ does not intersect $S$, and thus we have proved that $V \backslash S$ satisfies the condition of " $p_{7}$-convexity" of DocquierGrauert $[\mathbf{D G}]$. It follows that $V \backslash S$ is Stein.

Lemma 2. Let $S$ be a nonempty locally pseudoconcave subset of finite Hausdorff $(2 n-2)$-measure of an $n$-dimensional complex manifold $\Omega$. Then $S$ is a pure $(n-1)$-dimensional complex analytic subset of $\Omega$.

Proof: The statement is local, so we can assume that $\Omega$ is a domain in $\mathbb{C}^{n}$. Let $a \in S$ and choose coordinates $(z, w), z=\left(z_{1}, \ldots, z_{n-1}\right)$ in $\mathbb{C}^{n}$ in such a way that $a=0$ and the set $S \cap\{z=0\}$ is finite or countable (this can be done in the obvious way). Then there exists a neighbourhood $V=V^{\prime} \times V_{n}$ of 0 such that the projection of $S \cap V$ into $V^{\prime}$ is proper. It follows that the fibres $S \cap V \cap\{z=c\}$ are finite for almost every $c \in V^{\prime}$. By the Oka-Nishino Theorem $[\mathbf{N}] S \cap V$ is then a complex analytic set of pure dimension $n-1$.

\section{References}

[C] E. M. Chirka, “Complex analytic sets," Nauka, Moscow, 1985. 
English transl., Kluwer Acad. Publishers, Dordrecht-Boston-London, 1989.

[DG] F. Docquier And H. Grauert, Levisches Problem und Rungescher Satz für Teilgebiete Steinscher Männigfaltigkeiten, Math. Ann. 140 (1960), 94-123.

[I] S. M. Ivashrovich, The Hartogs-type extension theorem for meromorphic maps into compact Kähler manifolds, Invent. Math. 109 (1992), 47-54.

[KS] J. KAJIWARA AND E. SAKAI, Generalization of Levi-Oka's theorem concerning meromorphic functions, Nagoya Math. J. 29 (1967), $75-84$.

[La] M. LAWREnCE, Polynomial hulls and geometric function theory of several complex variables, Thesis, University of Washington, Seattle (1991).

[Lu] G. Lupacciolu, Meromorphic continuation in projective space, Bollettini U. M. I. (7) 4-A (1990), 391-397.

[N] T. Nishino, Sur les ensembles pseudoconcaves, J. Math. Kyoto Univ. 1-2 (1962), 225-245.

[R] R. Remmert, Holomorphe und meromorphe Abbildungen komplexer Räume, Math. Ann. 133 (1957), 328-370.

[S] E. L. Stout, Removable sets for holomorphic functions of several complex variables, Publ. Math. 33 (1989), 345-362.

[St] W. Stoll, Über meromorphe Modifikation, II, Math. Z. 61 (1955), 467-488.

Keywords. meromorphic mappings, removable singularities, pseudoconcave sets, analytic varieties.

1991 Mathematics subject classifications: Primary 32D15, 32D20; Secondary 32D10.

\author{
Steklov Mathematical Institute \\ Vavilov st. 42 \\ Moscow GSP-1 \\ 117966 RUSSIA \\ email: chirka@class.mian.su
}

Rebut el 4 de Desembre de 1995 